\title{
In-vivo-Diagnostik von Malignomen mit Kontakt-Endoskopie
}

\begin{abstract}
Wie effektiv ist die nicht-invasive Kontakt-Endoskopie zur frühzeitigen Erfassung von Plattenepithelkarzinomen? Kollegen der Universität Hannover gingen dieser Frage in einer prospektiven Studie nach.
\end{abstract}

D ie Kontakt-Endoskopie wurde zum ersten Mal 1979 beschrieben. Das Verfahren, mit dem man in vivo und in situ Schleimhaut auf benigne oder maligne Veränderungen untersuchen kann, beruht auf der Anfärbung oberflächlicher Epithelschichten mit Methylenblau. Die Endoskopspitze wird dann direkt auf die Schleimhaut aufgesetzt und das spezifische Farbmuster beurteilt.

Bei 42 Patienten mit verdächtigen Läsionen im Bereich von Pharynx, Larynx oder des Ösophagus erfolgte unter Allgemeinanästhesie zunächst eine Untersuchung mit dem Endoskop. Die mit Methylenblau angefärbten Regionen wurden mit 150-facher Vergrößerung beurteilt und fotografiert. Danach ent- nahmen die HNO-Ärzte bei den Patienten Biopsien und führten die Präparate einer histopathologischen Untersuchung als Referenzmethode zu.

32 der 42 Präparate $(76,2 \%)$ ergaben in der pathologischen Beurteilung einen benignen Befund. In einem Fall handelte es sich um unauffällige Schleimhaut, in 24 Fällen lag eine chronische Entzündung vor und sieben Präparate wurden als dysplastische Mukosa eingestuft. In zehn Fällen $(23,8 \%)$ stellten die Pathologen die Diagnose eines Plattenepithelkarzinoms.

Die Aufnahmen mit dem Endoskop wurden zunächst von einem in der Kontaktendoskopie unerfahrenen Zytopathologen beurteilt. Dabei identifizierte dieser
$90,6 \%$ der Befunde korrekt als benigne ( 29 von 32 Präparaten). Jedoch stufte er nur bei 70\% die Läsionen richtig als maligne ein (sieben von zehn Präparaten).

Der in der Kontakt-Endoskopie erfahrene HNO-Arzt dagegen beurteilte 93,8\% der benignen Veränderung richtig und erkannte in $90 \%$ der Fälle korrekt die Karzinome. Damit lagen für den geübten Kollegen bei der Erkennung von Malignomen die Sensitivität bei $90 \%$ und die Spezifität bei 93,8\%.

Fazit: In der Hand eines erfahrenen Facharztes zeigte die Kontakt-Endoskopie relativ hohe Werte für Sensitivität und Spezifität bei der Malignom-Diagnostik. Die Autoren weisen jedoch darauf hin, dass dieses nicht-invasive Verfahren die klassische Histopathologie nicht ersetzen kann.

Warnecke A et al. Contact endoscopy for the evaluation of the pharyngeal and laryngeal mucosa. Laryngoscope 2010; 120 : 253-8

\section{Dreifach-Kombination vor der Radiochemotherapie einsetzen?}

Bei fortgeschrittenem Nasopharynxkarzinom besteht die Standardtherapie aus einer synchron durchgeführten Radiochemotherapie mit oder ohne adjuvanter Chemotherapie. Nun wurde erstmals untersucht, ob eine Induktionstherapie mit der Dreifachkombination aus Docetaxel, Cisplatin und 5-Fluorouracil (5-FU) für Patienten mit lokoregional fortgeschrittenem Nasopharynxkarzinom eine Option ist.

B isher war nicht geklärt, ob bei Patienten mit Nasopharynxkarzinom die Chemotherapie vor oder nach der Radiochemotherapie effektiver ist. Die Chemotherapie wird aber möglicherweise vor der simultanen Radiochemotherapie besser toleriert als nachher.

In der vorliegenden Studie erhielten 33 Patienten mit bisher unbehandeltem fortgeschrittenem Nasopharynxkarzinom in den Stadien III oder IV drei Zyklen einer Induktionschemotherapie aus Docetaxel $\left(70 \mathrm{mg} / \mathrm{m}^{2}\right.$, Tag 1), Cisplatin $\left(75 \mathrm{mg} / \mathrm{m}^{2}\right.$, Tag 1) und 5 -FU $\left(1.000 \mathrm{mg} / \mathrm{m}^{2}\right.$, kontinuierliche Infusion an den Tagen 2-5). Danach wurde Cisplatin $\left(100 \mathrm{mg} / \mathrm{m}^{2}\right)$ alle drei Wochen zusammen mit der Radiotherapie verabreicht.

Nach der Induktionschemotherapie hatten 15,2\% der Patienten eine vollständige Remission und 81,8\% eine partielle Remission. Bei einem Patienten kam es zur Krankheitsprogression. Sechs Wochen nach der Radiochemotherapie hatten 69,7\% der Patienten eine vollständige Remission und 27,3\% eine partielle Remission.

Das progressionsfreie 3-Jahres-Überleben betrug 75,6\% und das 3-JahresGesamtüberleben 86,1\%. Die Lebensverlängerung ist vermutlich auf das seltenere Auftreten von Fernmetastasen zurückzuführen.
Die Dreifach-Chemotherapie wurde relativ gut toleriert. Die Grad-3/4-Toxizitäten waren eine Neutropenie (72,7\%), febrile Neutropenie $(9,1 \%)$ und Nausea (9,1\%). Während der Radiochemotherapie traten Mukositis (39,4\%), Fatigue $(15,2 \%)$ und Nausea $(9,1 \%)$ als häufigste Toxizitäten der Grade 3-4 auf. Zu Todesfällen kam es während oder kurz nach der Behandlung nicht.

Fazit: Die Induktion mit einer Dreifachkombination (Docetaxel, Cisplatin und 5-FU) und die nachfolgende Radiochemotherapie besitzt bei Patienten mit einem Nasopharynxkarzinom eine vielversprechende Anti-Tumor-Aktivität mit überschaubarer Toxizität. Damit könnte diese Behandlung sogar einen kurativen Ansatz bei fortgeschrittenem Nasopharynxkarzinom darstellen.

jn

Bae WK et al. Phase Il study of docetaxel, cisplatin, and 5-FU induction chemotherapy followed by chemoradiotherapy in locoregionally advanced nasopharyngeal cancer. Cancer Chemother Pharmacol 2010; 65: 589-95 